[5] Borgers, D., und Hueck, C.: Tabakmulties (TTC's) und Gesundheitspolitik, Jahrbuch für Kritische Medizin 8 (1982) 64-83.

[6] Helmert, U.: Analyse nationaler Trends kar diovaskulärer Risikofaktoren für die Bundesrepublik Deutschland von 1984 bis 1991 (eingereicht zur Publikation).

[7] Junge, B: Tabak, Jahrbuch Sucht. Geesthacht: Neuland (1995) 64-84.

[8] Borgers, D., und Menzel, R.: Wer raucht am meisten? MMW 126 (1984) 1092-1096.

[9] Borgers, D.: Rauchen und Berufe-Rauchverhalten bei 125 ausgewählten Berufen, Prävention 11 (1988) 12-15

[10] Brückner, G.: Gesundheitsrisiko »Rauchen «. Ergebnis des Mikrozensus 1989 zu den
Rauchgewohnheiten der Bevökerung. Wirtschaft und Statistik (1991) 341-352.

[11] Emmerling, D., und Riede, T.: 40 Jahre Mikrozensus. Wirtschaft und Statistik 3 (1997) 160-174.

[12] Macht, A.: Klassifizierung der Berufe 1992. Wirtschaft und Statistik (1992) 855-863.

[13] Blossfeld, H. P.: Kohortendifferenzierung und Karriereprozeß. Eine Längsschnittstudie über die Veränderung der Bildungs- und $\mathrm{Be}$ rufschancen im Lebenslauf. Frankfurt: Campus (1989).

[14] Statistisches Bundesamt, Fachserie 1, Reihe 4.1.1, Wiesbaden (1978).

[15] Beaglehole, R., and Bonita, B.: Public health at the crossroads. Cambridge: University Press, 1997.
[16] Rosén, M., Hanning, M., and Wall, S.: Changing smoking habits in Sweden: towards better health, but not for all. Int J Epidemiol 19 (1990) 316-322.

[17] Cavelaars, A. E. J. M., Kunst, A. E., Geurts, J. J. M., et al.: Socio-economic differences in smoking in 12 European countries (1997, eingereicht zur Veröffentlichung)

Anschrift der Verfasser:

Dr. Uwe Helmert, Abt. Gesundheitspolitik, Arbeits- und Sozialmedizin, Zentrum für Sozialpolitik, Universität Bremen, Parkallee 39, 28209 Bre men; PD Dr. med. Dieter Borgers, Dezernat Epidemiologie, Landesinstitut für den Öffentlichen Gesundheitsdienst Nordrhein-Westfalen, Westerfeldstr. 35-37, 33611 Bielefeld

\title{
Zur demographischen Situation in der Bundesrepublik Deutschland
}

Die jeweilige demographische Situation in einer Bevölkerung übt einen wesentlichen Einfluß auf die Häufigkeit von Krankheiten und Todesursachen aus. So hat der Altersaufbau einer Bevölkerung gravierende Auswirkungen auf das Sterbegeschehen. Die Sterbehäufigkeit an Herz-Kreislaufkrankheiten z. B. steigt mit zunehmendem Alter an. Je mehr Personen sich im höheren Lebensalter befinden, um so höher ist auch die Gesamtsterblichkeit an Herz-Kreislaufkrankheiten. Oder betrachtet man die Unfälle: Je mehr Personen in der Altersgruppe 15 bis 25 Jahre vorhanden sind, das ist die Altersgruppe mit der höchsten Unfallsterblichkeit, um so höher ist die Gesamtsterblichkeit an Unfällen. Der Anstieg oder der Rückgang einer Sterbeziffer bedeutet also nicht $z$ wangsläufig eine Steigerung oder Senkung des Sterberisikos an einer bestimmten Krankheit. Andererseits wirkt die Medizin mittelbar und unmittelbar auf demographische Prozesse ein. Die Zahl und die Häufigkeit von Gestorbenen werden von den Möglichkeiten der Medizin mit beeinflußt. Als Beispiele seien die Senkung der Säuglings- und Kindersterblichkeit sowie der Rückgang der Sterblichkeit an chronischen Krankheiten im höheren Lebensalter genannt. Die Kenntnis demographischer Daten ist darüber hinaus für gesundheitspolitische Entscheidungen unerläßlich; so erfordert beispielsweise die große und steigende Anzahl älterer Personen besondere Leistungen hinsichtlich ihrer medizinischen und sozialen $\mathrm{Be}$ treuung.

Im folgenden werden der Altersaufbau der Bevölkerung und die natürliche Bevölkerungsbewegung überblicksweise betrachtet.

\section{Bevölkerungsbestand}

Die Bundesrepublik Deutschland hatte am 31. 12. 1995 eine Wohnbevölkerung von 81,8 Millionen, davon waren 39,8 Millionen männlichen und 42 Millionen weiblichen Geschlechts [1]. Die Einwohnerzahl in den alten Bundesländern (frühere Bundesrepublik) betrug zu diesem Zeitpunkt 66,3 Millionen, in den neuen Bundesländern (ehemals DDR) 15,5 Millionen. Der Anteil der Ausländer an der Gesamtbevölkerung macht etwa $9 \%$ aus. Aus der Einwohnerzahl resultiert eine Bevölkerungsdichte von 266 Einwohnern/qkm in den alten und von 131 Einwohnern/qkm in den neuen Bundesländern.

\section{Altersaufbau der Bevölkerung}

Der Altersaufbau der Bevölkerung läßt sich graphisch darstellen in einem sogenannten Lebensbaum (Abb. 1). In der Senkrechten sind die Altersjahre aufgetragen und in der Waagerechten die Anzahl der Personen im jeweiligen Alters- jahr getrennt nach dem Geschlecht. Bis zum Alter von 40 Jahren ist ein Überschuß männlicher Personen und ab etwa 65 Jahre ein deutlicher Überschuß weiblicher Personen zu erkennen. Der Frauenüberschuß ist hauptsächlich bedingt durch die höhere Sterblichkeit der Männer jenseits des mittleren Lebensalters und durch die Auswirkungen der großen Kriege in unserem Jahrhundert, von denen insbesondere im Zweiten Weltkrieg die Zivilbevölkerung ebenfalls betroffen war. Der Männerüberschuß im jüngeren Alter wird begründet durch das Geschlechtsverhältnis bei der Geburt; es werden durchschnittlich 105 Knaben je 100 Mädchen geboren. Der Männerüberschuß wird sich künftig erhöhen infolge der intensiveren Senkung der männlichen Säuglingssterblichkeit sie ist bekanntlich bei männlichen Säuglingen höher als bei weiblichen. Eine Senkung der männlichen Sterblichkeit an nichtnatürlichen Todesursachen wie Unfall, Suizid, Gewalteinwirkungen wird ebenfalls dazu beitragen.

Der Altersaufbau der deutschen Bevölkerung ist nach wie vor von starken Unregelmäßigkeiten geprägt. Die beiden Weltkriege haben deutliche Spuren hinterlassen. Die tiefen Einschnitte im Alter um 80 Jahre kennzeichnen den Geburtenausfall während des ersten Weltkrieges, die Einschnitte um das 50. Jahr sind sowohl Auswirkungen des Ge- 


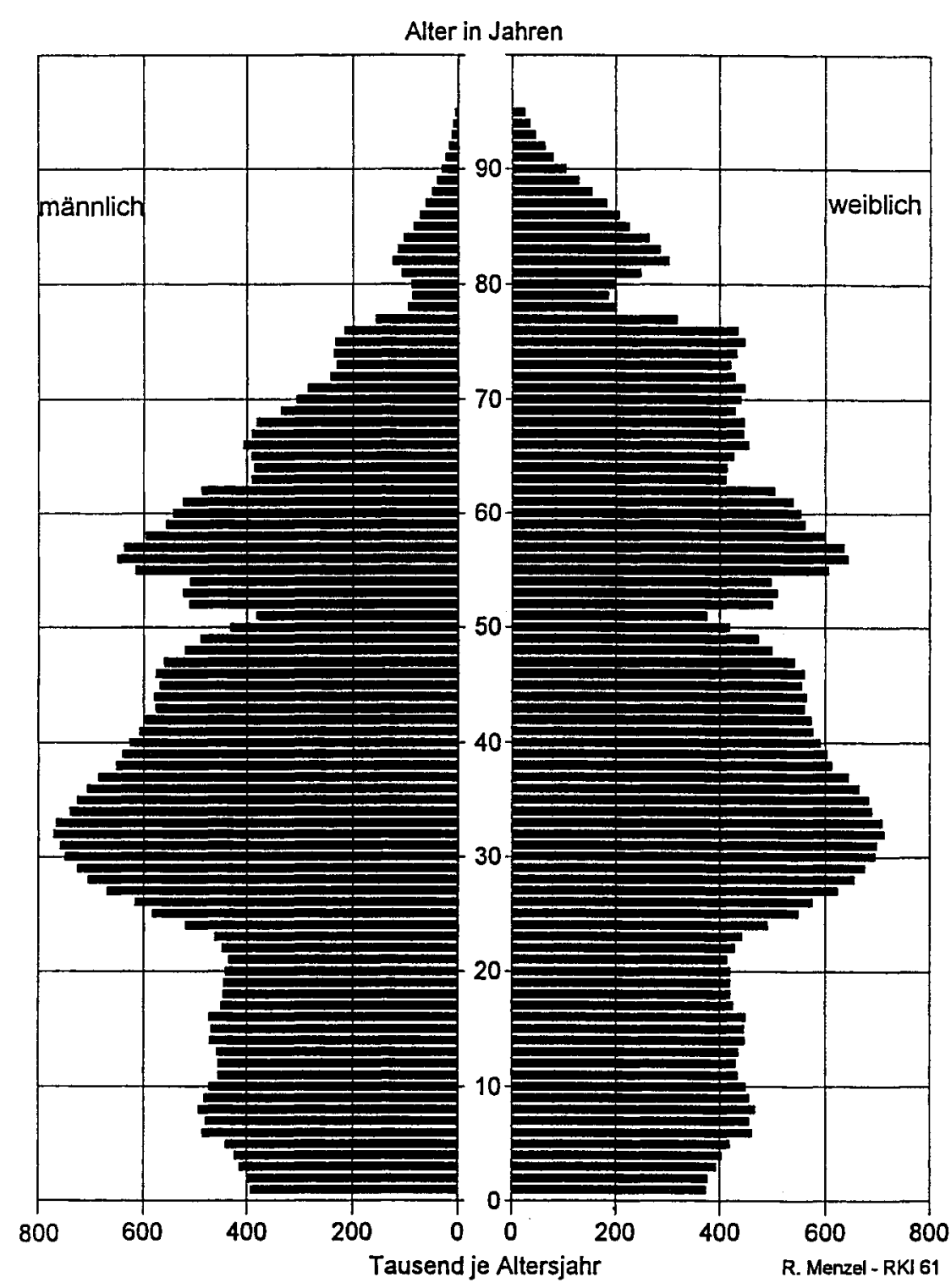

Abbildung 1: Altersaufbau der Bevölkerung am 31.12.1995 - Bundesrepublik Deutschland. nannter Faktor den größeren Anteil am Geburtenrückgang. Die Einschnitte im Lebensbaum wiederholen sich jeweils nach einer Generation und sind über mehrere Generationen als Auswirkungen bereits vorhandener Einschnitte zu erkennen. Man spricht in diesem $\mathrm{Zu}$ sammenhang vom »Demographischen Echo«.

Im Jahr 1995 ist der Anteil der Kinder (bis zum Alter von 14 Jahren) an der Gesamtbevölkerung mit 16,2 \% geringfügig höher als der Anteil der Personen im Alter von 65 Jahren und darüber, der $15,5 \%$ beträgt. Die höheren Anteile der Kinderbevölkerung sowie der Altersgruppe 15 bis 24 Jahre in den neuen Bundesländern resultieren noch aus den höheren Geburtenziffern in der DDR.

Ein Überblick über die Zusammensetzung der Bevölkerung nach Altersgruppen ist der Tabelle $1 \mathrm{zu}$ entnehmen [1].

Der Anteil der 65jährigen und älteren Personen an der Gesamtbevölkerung ist im Zeitraum von 1980 bis 1995 in den alten und neuen Bundesländern sowohl bei den Männern als auch bei den Frauen leicht angestiegen, er liegt 1995 bei $15,7 \%$ im westlichen und bei $14,6 \%$ im östlichen Landesteil (Abb. 2). Für das Jahr 2010 wird dieser Anteil bei 19,9\% in den alten und bei $19,8 \%$ in den neuen Bundesländern prognostiziert [2]. Von 100 der über 65jährigen sind 41 Personen älter als 75 Jahre und elf Personen älter als 85 Jahre. Im Jahre 1995 waren in der Bundesrepublik insgesamt 53500 Personen 95 Jahre und älter.

Medizinische Fortschritte, vor allem in der Bekämpfung chronischer Krankheiten, und eine allgemeine Verbesserung der Lebensbedingungen alter Menschen führen zu einem Rückgang der Sterb- burtenausfalls des ersten Weltkrieges (die Zahl der Geborenen wird von der Zahl der Frauen im gebärfähigen Alter bestimmt) als auch direkter Geburtenausfall während des zweiten Weltkrieges. Der drastische Rückgang der Bevölkerungszahl vom 20. Lebensjahr an abwärts hat mehrere Gründe: Einerseits zeigen sich die Auswirkungen des Geburtenausfalls während des zweiten Weltkrieges, andererseits vollzog und vollzieht sich ein deutlicher Wandel im generativen Verhalten der Bevölkerung, der einen allgemeinen Rückgang der Fruchtbarkeit zur Folge hat. Nach den Berechnungen des Bundesinstitutes für Bevölkerungsforschung hat letztge-
Tabelle 1: Bevölkerung nach Altersgruppen absolut sowie Anteile in Prozent der jeweiligen Bevölkerung getrennt nach Geschlecht in den alten und neuen Bundesländern

\begin{tabular}{|c|c|c|c|c|c|c|}
\hline \multirow[b]{3}{*}{ Altersgruppen } & \multicolumn{6}{|c|}{ Bevölkerung } \\
\hline & \multicolumn{2}{|c|}{ alte Bundesländer } & \multicolumn{2}{|c|}{ neue Bundesländer } & \multicolumn{2}{|c|}{ Deutschland ges. } \\
\hline & $\begin{array}{l}\text { absolut } \\
\text { in } 1000\end{array}$ & $\begin{array}{c}\text { Anteile } \\
\text { in } \%\end{array}$ & $\begin{array}{l}\text { absolut } \\
\text { in } 1000\end{array}$ & $\begin{array}{l}\text { Anteile } \\
\text { in } \%\end{array}$ & $\begin{array}{l}\text { absolut } \\
\text { in } 1000\end{array}$ & $\begin{array}{c}\text { Anteile } \\
\text { in } \%\end{array}$ \\
\hline unter 14 Jahre & 10684 & 16,2 & 2579 & 16,6 & 13264 & 16,2 \\
\hline 15-24 Jahre & 7364 & 11,1 & 1862 & 12,0 & 9226 & 11,3 \\
\hline 25-44 Jahre & 21203 & 32,1 & 4861 & 31,4 & 26064 & 31,9 \\
\hline 45-64 Jahre & 16533 & 24,9 & 3940 & 25,4 & 20474 & 25,1 \\
\hline über 65 Jahre & 10372 & 15,7 & 2262 & 14,6 & 12634 & 15,5 \\
\hline zusammen & 66156 & 100 & 15505 & 100 & 81661 & 100 \\
\hline
\end{tabular}




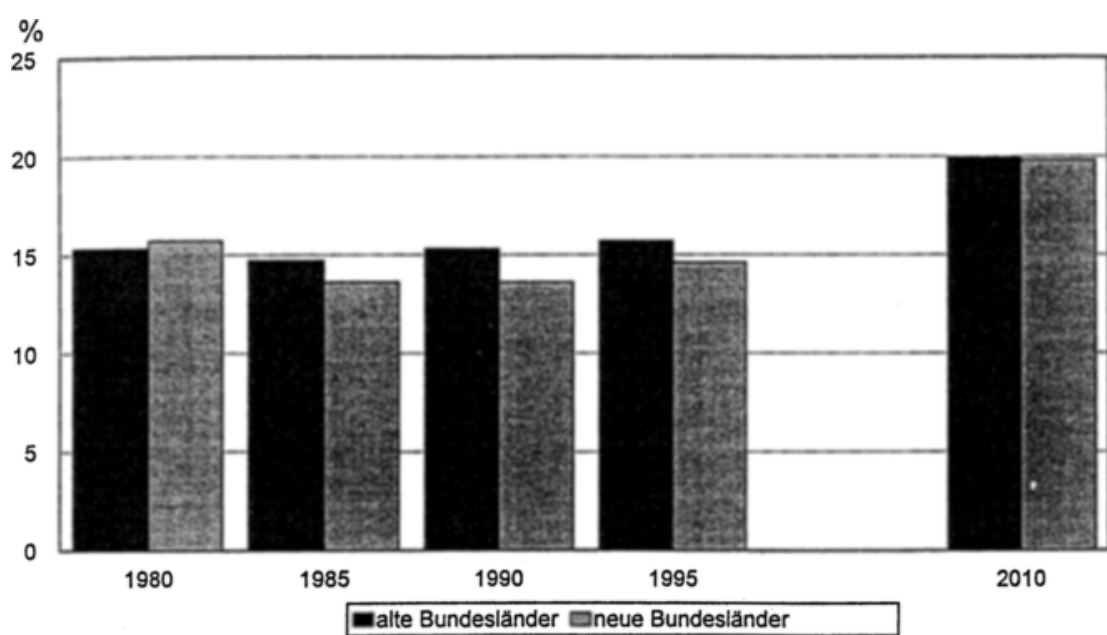

Abbildung 2: Anteil der 65jährigen und älteren Personen an der Wohnbevölkerung 1980 bis 1995 und Prognose für 2010 - alte und neue Bundesländer.

lichkeit. Immer mehr Menschen erreichen ein hohes Alter. Weil damit die Zahl der Personen mit chronischen und behindernden Krankheiten wächst, hat das Altern der Bevölkerung erhebliche Auswirkungen für die Planung und Organisation des Gesundheitswesens. Multimorbidität und Pflegebedürftigkeit und die dadurch entstehenden Anforderungen an die Betreuung stellen das Gesundheitswesen und auch andere gesellschaftliche Bereiche vor große Aufgaben. Nach internationalen Studien wird eine behinderungsfreie Lebenszeit auf etwa 60 Jahre für männliche Personen und auf etwa 64 Jahre für weibliche Personen geschätzt [3]. Verläßliche Daten aus Deutschland liegen dazu nicht vor. Die mittlere Lebenserwartung beträgt gegenwärtig bei den männlichen Personen 73 Jahre und bei den weiblichen Personen 79 Jahre. Sie ist seit 1980 um durchschnittlich drei Jahre gestiegen. Studien deuten darauf hin, daß im Verlauf des Aging-Prozesses aber auch mehr Menschen ein höheres Alter ohne Behinderungen erreichen [4, 5]. Diese Entwicklung dürfte sich auch in der Bundesrepublik Deutschland vollziehen.

\section{Fruchtbarkeit und Bevölke- rungsbilanz}

Im Jahre 1995 wurden in Deutschland 765200 Lebendgeborene registriert, 681400 in den alten und 83800 in den neuen Bundesländern.

Für die Beurteilung des generativen Verhaltens einer Bevölkerung bezieht man die Zahl der Lebendgeborenen auf die Zahl der Frauen im gebärfähigen (fertilen) Alter, das ist die Altersspanne von 15 bis 45 Jahre. Die so ermittelte Ziffer ist die allgemeine Fruchtbarkeitsoder Fertilitätsziffer. Im Jahre 1995 haben 1000 Frauen im fertilen Alter in den alten Ländern im Durchschnitt 49 und in den neuen Ländern im Durchschnitt 26 Kinder lebend geboren. In den alten Bundesländern variieren die allgemeinen Fruchtbarkeitsziffern im betrachteten Zeitraum von 1980 bis 1995 nur geringfügig, in den neuen Bundesländern sind sie gemessen an 1980 auf etwa ein Drittel zurückgegangen, wobei der Rückgang von $1989 \mathrm{zu} 1990 \mathrm{mehr}$ als $50 \%$ beträgt (Abb. 3); die absolute Zahl der Lebendgeborenen ist um 115000 gesunken $[1,6]$.

Betrachtet man die altersspezifischen Fruchtbarkeitsziffern, d. h. die Lebendgeborenenhäufigkeiten nach dem Alter der Mutter, ist zu erkennen, daß die Differenz in den allgemeinen Fruchtbarkeitsziffern zwischen den alten und den neuen Bundesländern vor allem durch die geringere Fruchtbarkeit in den Altersgruppen ab 27 Jahre im Osten verursacht wird (Abb. 4). Das Alter höchster Fruchtbarkeit liegt in den alten Ländern bei 29 Jahren, in den neuen bei 25 Jahren.

Unter der Voraussetzung, daß die Fruchtbarkeitsverhältnisse des Jahres 1995 fortbestehen und keine Frauen im gebärfähigen Alter sterben, würden 1000 Frauen im Verlaufe ihres fertilen Alters in den alten Bundesländern 1346 und in den neuen 772 Kinder gebären (je Frau demnach 1,3 resp. 0,8 Kinder). Diese Ziffer ist die Summe der altersspezifischen Fruchtbarkeitsziffern und wird als Gesamtfruchtbarkeitsziffer (auch zusammengefaßte Geburtenziffer) bezeichnet. Im Jahre 1990 betrug sie in beiden Landesteilen noch etwa 1,5 [2].

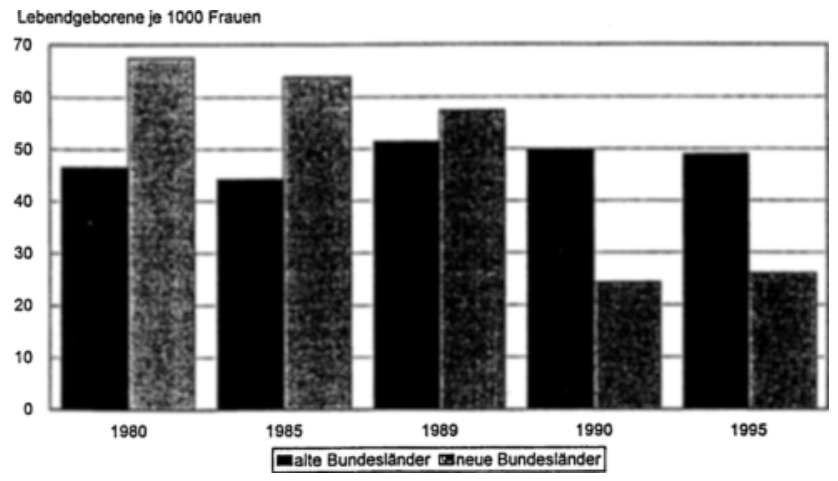

Abbildung 3: Allgemeine Fruchtbarkeitsziffern (Lebendgeborene je 1000 Frauen im Alter von 15 bis 45 Jahre) 1980 bis 1995 - alte und neue Bundesländer.

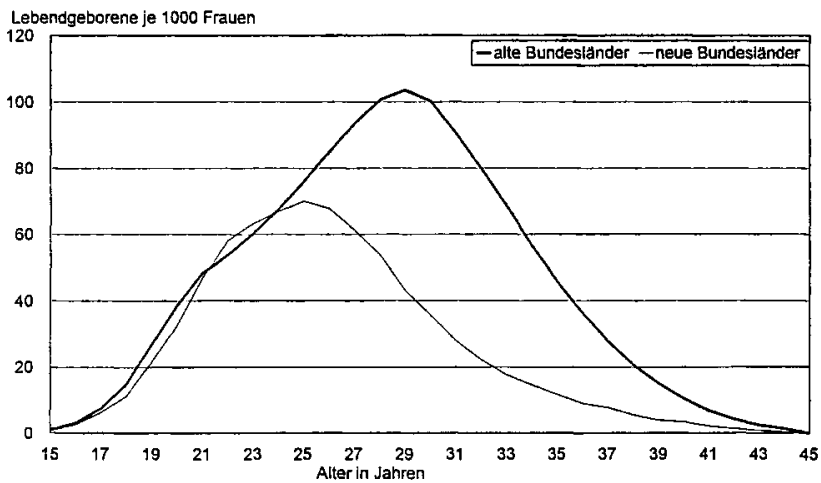

Abbildung 4: Altersspezifische Fruchtbarkeitsziffern (Lebendgeborene je 1000 Frauen des jeweiligen Alters) 1995 - alte und neue Bundesländer. 

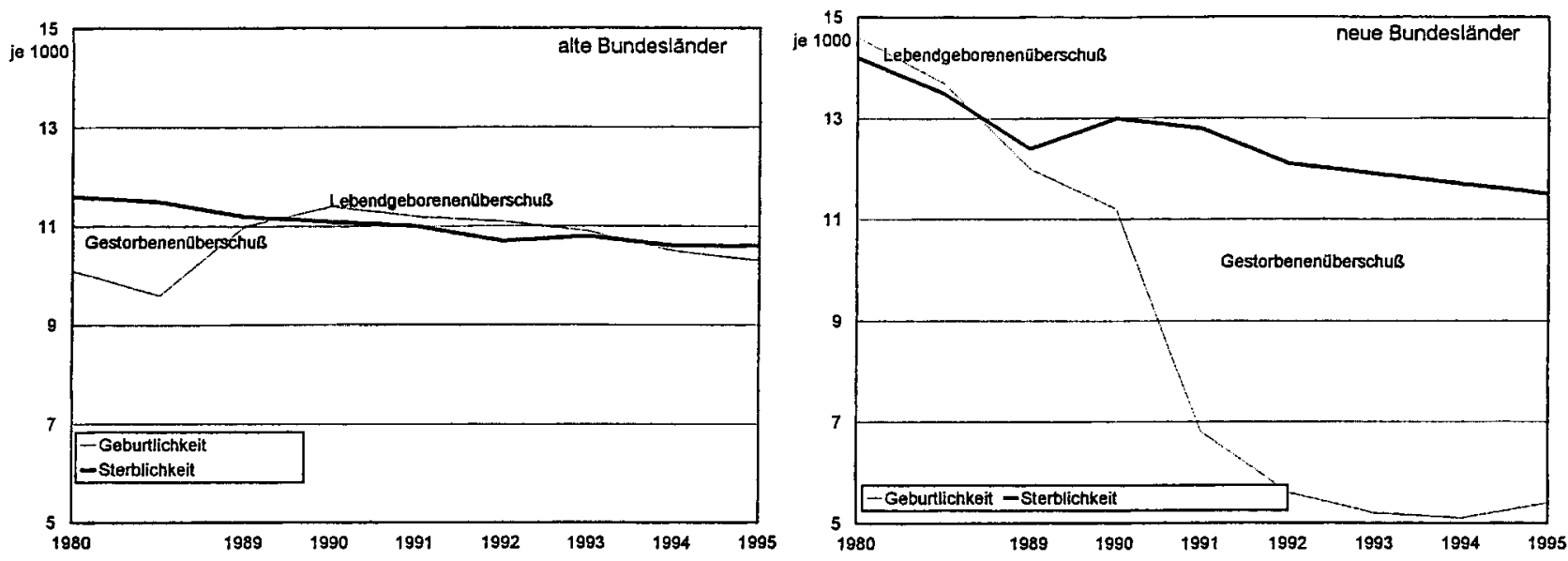

Abbildung 5: Geburtlichkeit, Sterblichkeit und Bevölkerungsbilanz 1980 und 1989 bis 1995 - Lebendgeborene/Gestorbene je 1000 der Bevölkerung.

\section{Bevölkerungsbilanz}

Die Entwicklung ( $\mathrm{Zu}$ - oder Abnahme) der Bevölkerungszahl eines Gebietes ist unmittelbar und vor allem von der natürlichen Bevölkerungsbewegung in diesem Gebiet abhängig, also von den Geburten und den Sterbefällen. Für die Bilanzierung werden die Lebendgeborenen und die Gestorbenen auf jeweils 1000 der Gesamtbevölkerung bezogen. Die Bilanz der natürlichen Bevölkerungsbewegung (Abb. 5) zeigt bis 1989 für die alten Bundesländer einen $\mathrm{Ge}$ storbenenüberschuß und verläuft mit leicht sinkender Tendenz beider Ziffern seit 1990 relativ ausgeglichen. Im Jahr 1995 besteht trotz der höheren Fertilität der Ausländer ein Gestorbenenüberschuß. In den neuen Bundesländern bestand bis etwa 1985 ein leichter Lebendgeborenenüberschuß, ab 1990 beginnt ein sich drastisch vergrößernder $\mathrm{Ge}$ storbenenüberschuß. Der geringfügige Anstieg der Lebendgeborenenhäufigkeit im Jahr 1995 und der ständige Rückgang der Gestorbenenhäufigkeit ändern wenig an der ungünstigen demographischen Situation im Osten Deutschlands.

Hinsichtlich der Bevölkerungszahl wird der Gestorbenenüberschuß 1995 in den alten Bundesländern durch Zuwanderungen kompensiert, so daß gegenüber dem Vorjahr ein Bevölkerungszuwachs um $0,5 \%$ zu verzeichnen ist. Die Bevölkerungszahl in den neuen Ländern ist um $0,4 \%$ gesunken.

\section{Resümee}

Die demographische Entwicklung in der Bundesrepublik Deutschland wird durch einen Fruchtbarkeitsrückgang, den die sinkende Sterblichkeit nicht ausgleichen kann, gekennzeichnet. Die einfache Bevölkerungsreproduktion ist zur Zeit nicht gegeben. Der abnehmenden Zahl jüngerer Personen steht eine sich ständig vergrößernde Anzahl älterer Personen gegenüber. Dieser Sachverhalt stellt ein gesundheits-, gesellschaftsund familienpolitisches Problem dar.

\section{Zusammenfassung}

Die demographische Situation einer Bevölkerung hat einen wesentlichen Einfluß auf die Häufigkeit von Todesursachen und Krankheiten. Ohne Kenntnis der demographischen Gegebenheiten sind Mortalität und Morbidität kaum gültig zu beurteilen. Darüber hinaus ist die Kenntnis demographischer Daten für gesundheitspolitische Entscheidungen unerläßlich. Die demographische Situation in der Bundesrepublik Deutschland ist geprägt durch einen Fruchtbarkeitsrückgang und einen Anstieg der Lebenserwartung. Der abnehmenden Zahl jüngerer Personen steht eine ständig steigende Zahl älterer Personen gegenüber. Dieser demographische Wandel führt erwartungsgemäß zu einem Häufigkeitsanstieg an chronischen und behindernden Krankheiten und ist somit eine gesundheits- und sozialpolitische Herausforderung. Andererseits er- reichen nicht nur mehr Menschen ein höheres Alter, sondern mehr Menschen erreichen dieses Alter auch in besserer Gesundheit, so daß auch die behinderungsfreie Lebenserwartung wächst.

\section{Literatur:}

[1] Statistisches Bundesamt: Statistisches Jahrbuch für die Bundesrepublik Deutschland 1995.

[2] Grünheid, E., und Schulz, R.: Bericht 1996 über die demographische Lage in Deutschland. Zeitschrift f. Bevölkerungswissenschaft, 21, 4 (1996) 345-439.

[3] Robine, J.-M.: Estimation de la valeur de l'espérance de vie sans incapacité (EVSI) pour les pays occidentaux au cours de la dernière décennie. Quelle peut être l'utilité de ce nouve indicateur de l'état de santé? Rapp. trimest. statist. sanit. mond. 42 (1989) 141-150, 1989.

[4] Guralnik, J. M., and Kaplan, G. A.: Predictors of Healthy Aging: Prospective Evidence from the Alameda County Study. AJPH June 1989, Vol. 79 , No. 6.

[5] Schneider, E. L., and Brody, J. A.: Aging, natural death and the compression of morbidity Another view. N. Engl. J. Med. 309 (1983) 854-855.

[6] Statistisches Bundesamt: Sonderreihe mit Beiträgen für das Gebiet der ehemaligen DDR. Heft 1: Bevölkerungsstatistische Übersichten.

\section{Weiterführende Literatur:}

- Casper, W., et al.: Demographische Situation. In: Das Gesundheitswesen der DDR. Jg. 20 (1985) bis 25 (1990). Institut für Medizinische Statistik und Datenverarbeitung, Berlin.

- Förster, E., und Giersdorf, P.: Grundlagen der Demographie. Schriftenreihe der Akademie für Ärztliche Fortbildung der DDR, H. 50. VEB Verlag Volk und Gesundheit, Berlin 1976.

- WHO: Fact Sheet 135/1996.

Dr. Waldtraut Casper, Fachbereich Nicht übertragbare Krankheiten und Gesundheitsberichterstattung, Robert Koch-Institut, Berlin 